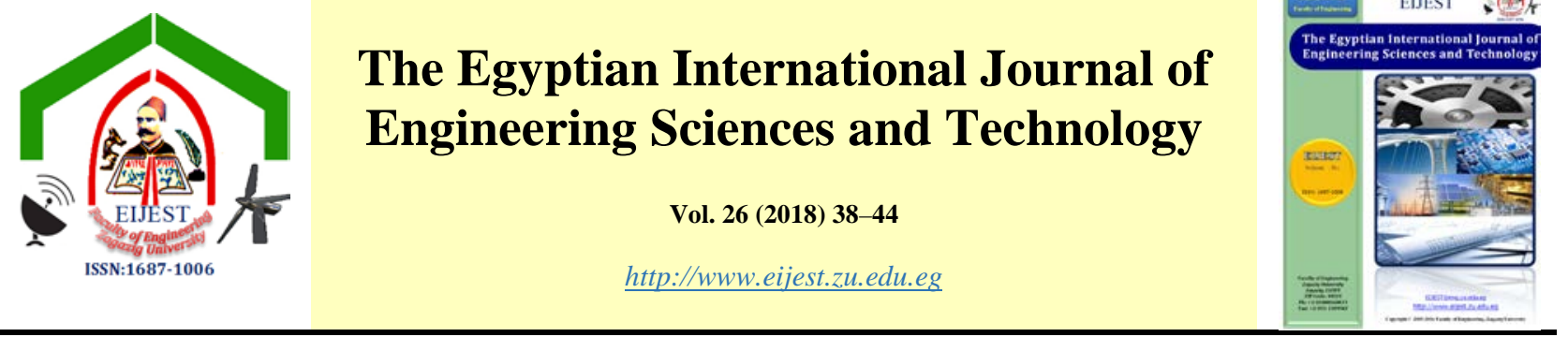

\title{
Fully Implicit Scheme for Solving Burgers' Equation based on Finite Difference Method
}

\author{
N. A. Mohamed*
}

Department of Engineering Mathematics and Physics, Faculty of Engineering, Zagazig University, P.O. 44519, Zagazig, Egypt.

\begin{tabular}{l}
\hline A R T I C L E I N F O \\
\hline Article history: \\
Received 18July 2018 \\
Received in revised form \\
12August 2018 \\
Accepted on 28 August 2018 \\
Available online \\
15November 2018 \\
\hline
\end{tabular}

Keywords:

Burgers' equation

nonlinear one-dimensional

finite difference method

kinematic viscosity

\section{Introduction}

In this work, we consider the one-dimensional time dependent Burgers' equation in the form:

$$
\frac{\partial u}{\partial t}+u \frac{\partial u}{\partial x}=v \frac{\partial^{2} u}{\partial x^{2}}, \quad(x, t) \in \Omega \times[0, T],(1)
$$

with the initial condition

$$
u(x, 0)=f(x), \quad 0 \leq x \leq 1(2)
$$

andDirichlet boundary conditions

$$
u(0, t)=u(1, t)=0 ; \quad 0 \leq t \leq T,
$$

where $\Omega=(0,1)$ and $v>0$ is kinematic viscosity coefficient. the structure of Burgers' equation is similar to the Navier-Stokes equation due to the combination of convection, diffusion and a time dependent terms. The Burgers equation (1) is one of the very few nonlinear partial differential equations which can be solved exactly for restricted set of initial functions $f(x)$ only. So, various numerical methods have been introduced by researchers for studying the properties of it due to its wide range of applicability in respective fields of science and engineering.

Burgers' equation was first introduced by Bateman [1] who studied its steady state solution. Later, describing a mathematical model of turbulence, it was proposed by Burgers and due to the extensive work of Burgers, therefore it is referred as “'Burgers' equation', In a series of papers [2, 3], Burgers investigated various aspects of turbulence and also studied the statistical and spectral aspects of the equation and related systems of equations. Burgers' equations have been solved analytically in Hopf [4] and Cole [5] for a restricted set of arbitrary initial conditions. Burgers' equation is studied by many researchers for many reasons:

* Corresponding author. Tel.: +201001562199.

E-mail address: Norhan.alla@gmail.com. 
- First, Its analytical solution was obtained by Cole [5] so it is easy to have the numerical comparison.

- Second, It contains the simplest form of nonlinear convection term and diffusionterm for simulating the physical phenomena of wave motion.

- Third, Its shock wave behavior when $v$ is very small.

Several numerical treatments of this equation have been presented bymany researchers.For instance, treatments based on the Finite difference method in $[6,7,8]$, Finite elements method in [9, 10], spectral least-squares method in $[11,12,13]$, B-splines collocation method in $[14,15,16]$, explicit and exact explicit finite difference methods in [17], variational iteration method in [18, 19], Adomian-Pade technique in [20], homotopy analysis method in [21, 22], spectral collocation method in [23, 24], polynomial based differential quadrature method in [25], B-spline differential quadrature method in [26, 27, 28], cubic Hermite collocation method in [29], hybrid numerical scheme based on Haar wavelets in [30], high order splitting method in [31], Higherorder accurate finite difference method in [32], etc.

In this paper, the nonlinear term in Burger's equation is treated with a numerical formula that is proposed in KAY [33]. The technique based on finite difference method and is used to solve the onedimensional burger's equation and compared with the most popular known explicit methods as Euler forward discretization (EF) and Mac Cormack discretization (MCOR).

\section{The Solution procedure}

The Burgers equation in (1) can be rewrittenas:

$$
\frac{\partial u}{\partial t}=-u \frac{\partial u}{\partial x}+v \frac{\partial^{2} u}{\partial x^{2}}, \quad P_{1} \leq x \leq P_{2}, t \in[0, T],(4)
$$

with initial and boundary conditions as in (2), (3) respectively. First, Semi-discretization is obtained by using forward finite differencediscretization along $t$ direction while $x$ direction remains undiscretized. Then, the full- discretizationis obtainedby using central finitedifferencediscretization along $x$ direction.

\subsection{Semi- discretization}

Divide the interval $[0, T]$ into $N$ steps $0=\mathrm{t}_{0} \leq$ $\mathrm{t}_{1} \leq \cdots \leq \mathrm{t}_{\mathrm{N}}=T, \Delta t=T / \mathrm{N}$ and $t_{n}=n * \Delta t$ for $n=$ $1,2, \ldots \ldots, \mathrm{N}$ and defining the current time step as $K_{n+1}=t_{n+1}-t_{n}$ and apply the forward finite differenceformula along $t$ direction in equation (4), it becomes

$$
\frac{1}{K_{n+1}} u^{n+1}-v u_{x x}^{n+1}+u^{n+1} u_{x}^{n+1}=\frac{1}{K_{n+1}} u^{n}
$$

From equation (5) the nonlinear term $u^{n+1} u_{x}^{n+1}$ is needed to compute at every time step. In this work the linearization of this term is done as in [33], such that $u^{n+1} u_{x}^{n+1} \approx w^{n+1} u_{x}^{n+1}$, where $w^{\mathrm{n}+1}$ is computed by linear extrapolation using $u^{n}$ and $u^{n-1}$ as:

$$
u^{\mathrm{n}+1} \cong w^{\mathrm{n}+1}=\left(1+\left(\frac{K_{n+1}}{K_{n}}\right)\right) u^{n}-\left(\frac{K_{n+1}}{K_{n}}\right) u^{n-1}
$$

Substitution of (6) in (5) yields,

$$
\begin{aligned}
& \frac{1}{K_{n+1}} u^{n+1}-v u_{x x}^{n+1}+\left(\left(1+\left(\frac{K_{n+1}}{K_{n}}\right)\right) u^{n}-\right. \\
& \left.\left(\frac{K_{n+1}}{K_{n}}\right) u^{n-1}\right) u_{x}^{n+1}=\frac{1}{K_{n+1}} u^{n}(7)
\end{aligned}
$$

On discretizing along $x$ direction, the Central Finite Difference discretization for the terms $u_{x x}^{n+1}$ and $u_{x}^{n+1}$ in equation (7) is used.

\subsection{Full- discretization}

Divide the interval $\left[P_{1}, P_{2}\right]$ into $\mathrm{M}$ steps $P_{1}=$ $\mathrm{x}_{0} \leq \mathrm{x}_{1} \leq \cdots \leq \mathrm{x}_{\mathrm{M}}=P_{2}$, with constant spacing step $h=\left(P_{2}-P_{1}\right) / \mathrm{M}$ and $x_{i}=i h$ for $i=1,2, \ldots ., \mathrm{M}$, given $u^{n}$ at time level $t_{n}$ and compute $u^{\mathrm{n}+1}$ at $t_{n+1}$ via 


$$
\begin{gathered}
\frac{1}{K_{n+1}} u_{i}^{n+1}-\frac{v}{h^{2}}\left[u_{i+1}^{n+1}-2 u_{i}^{n+1}+u_{i-1}^{n+1}\right]+ \\
\frac{1}{2 h}\left(\left(1+\left(\frac{K_{n+1}}{K_{n}}\right)\right) u_{i}^{n}-\left(\frac{K_{n+1}}{K_{n}}\right) u_{i}^{n-1}\right)\left[u_{i+1}^{n+1}-u_{i-1}^{n+1}\right]= \\
\frac{1}{K_{n+1}} u_{i}^{n}(8)
\end{gathered}
$$

In this work, equal time step is used such that $K_{n}=$ $K_{n+1}=\Delta t$, so equation (8) will be written as:

$$
\begin{array}{r}
{\left[\frac{1}{\Delta t}+\frac{2 v}{h^{2}}\right] u_{i}^{n+1}+\left[-\frac{v}{h^{2}}+\frac{1}{2 h}\left(2 u_{i}^{n}-u_{i}^{n-1}\right)\right] u_{i+1}^{n+1}+} \\
{\left[-\frac{v}{h^{2}}-\frac{1}{2 h}\left(2 u_{i}^{n}-u_{i}^{n-1}\right)\right] u_{i-1}^{n+1}=\left[\frac{1}{\Delta t}\right] u_{i}^{n}(9)}
\end{array}
$$

Multiplication of equation (14) by $\Delta t h^{2}$ yields :

$$
\begin{gathered}
{\left[h^{2}+2 v \Delta t\right] u_{i}^{n+1}+\left[-v \Delta t+\frac{1}{2} \Delta t h\left(2 u_{i}^{n}-u_{i}^{n-1}\right)\right] u_{i+1}^{n+1}} \\
+\left[-v \Delta t-\frac{1}{2} \Delta t h\left(2 u_{i}^{n}-u_{i}^{n-1}\right)\right] u_{i-1}^{n+1}=h^{2} u_{i}^{n}(10)
\end{gathered}
$$

Equation (10) can be written in a simple form as:

where,

$$
\alpha_{i} u_{i}^{n+1}+\beta_{i} u_{i+1}^{n+1}+\gamma_{i} u_{i-1}^{n+1}=f_{i}(
$$

$$
\begin{gathered}
\alpha_{i}=h^{2}+2 v \Delta t \\
\beta_{i}=-v \Delta t+\frac{1}{2} \Delta t h\left(2 u_{i}^{n}-u_{i}^{n-1}\right) \\
\gamma_{i}=-v \Delta t-\frac{1}{2} \Delta t h\left(2 u_{i}^{n}-u_{i}^{n-1}\right) \\
f_{i}=h^{2} u_{i}^{n}
\end{gathered}
$$

Apply equation (11) at every point $i$, then solve the system of equations $B u=F$ at every time step to find $u$ of all point at every time step.

\section{The Numerical Results}

Burgers' equation in (1-3) is solved by the proposed method and the numerical results are compared with the exact solution and with other known explicit methods i.e., Euler forward discretization (EF) and Mac Cormack discretization (MCOR) at different nodal points and at different values of kinematic viscosity $v$.

Since the exact solution is given, the $\mathrm{L}_{2}$ discretization error norm is computed after each time step by using the following definitions:

$$
\begin{aligned}
L_{2}:=\| u_{\text {exact }}- & u_{\text {computed }} \|_{2} \\
& =\sqrt{\frac{\left(\sum_{j=1}^{n}\left|u_{j}^{\text {exact }}-u_{j}^{\text {computed }}\right|^{2}\right)}{N}}
\end{aligned}
$$

The discretization error norms at the last time step are listed and compared with Euler forward (EF) and Mac Cormack discretization (MCOR).

Example 1:

Solve Burgers' equation in (1-3) with Initial condition:

$$
u(x, 0)=f(x)=\frac{2 v \pi \sin (\pi x)}{a+\cos (\pi x)}, \quad a>1
$$

whose exact solution is given by Wood [34]:

$$
u(x, t)=f(x)=\frac{2 v \pi e^{-v \pi^{2} t} \sin (\pi x)}{a+e^{-v \pi^{2} t} \cos (\pi x)}
$$

Numerical results obtained by the proposed method for $a=1.1, h=0.0125, T=1, v=0.1$ and time step $\Delta t=0.01$ are listed at different nodal points in Table (1). Table (2) reports the comparison of the proposed method with other known explicit methods, (EF) and (MCOR), to get $\mathrm{L}_{2}-$ norm for $a=1.1, h=0.0125, T=$ 1,10 for $v=0.01,0.1,1$. To investigate the stability of proposedmethod, the $\mathrm{L}_{2}$ - error norms at the last time step are listed in Table (3) for different values of initial condition parameter: $a$ and viscosity $v$.These results show that the proposed method is stable for a wide range of $v$. As mention above, other known numerical methods converges only within limited range of $v$.

Fig. 1 showed the numerical solution obtained by the proposed method and exact solution for $a=1.1, h$ $=0.0125, T=1, \Delta t=0.001$ and $v=0.01$.It is cleared that the numerical solution is almost identical to the exact solution. Fig. 2 showed the effect of increasing the coefficient a on the decreasing of $\mathrm{L}_{2}-$ error norm for the values of $v=1$, 10.In Fig.3, $\mathrm{L}_{2}-$ error norm is shown for $a=1.1, h=0.0125, \Delta t=0.001$ and $T=1$ for different value of $v$. It is cleared that $\mathrm{L}_{2}-$ error norm decreased as $v$ increased. It is clearedfrom Fig. 2 and Fig. 3 that decreasing the value of $v$, needs more of the time iterations to reduce the error norm. 
Table 1. Comparison of the proposed method with exact solution at different space points and the $\mathrm{L}_{2}$ - error norm for $a=1.1, h=0.0125, T=1,10$ for $v=0.1$.

\begin{tabular}{ccccc}
\hline$x$ & $\begin{array}{c}\text { Proposed } \\
\text { Method }\end{array}$ & $\begin{array}{c}\text { Exact } \\
\text { Solution }\end{array}$ & $\begin{array}{c}\text { Proposed } \\
\text { Method }\end{array}$ & $\begin{array}{c}\text { Exact } \\
\text { Solution }\end{array}$ \\
\cline { 2 - 5 } & \multicolumn{1}{c}{$\Delta t=0.001$} & $\Delta t=0.01$ \\
\hline 0.1 & 0.049787 & 0.049754 & $9.6339 \mathrm{e}-06$ & $9.12930 \mathrm{e}-06$ \\
0.2 & 0.098285 & 0.098212 & $1.8325 \mathrm{e}-05$ & $1.73650 \mathrm{e}-05$ \\
0.3 & 0.143751 & 0.143627 & $2.5222 \mathrm{e}-05$ & $2.39011 \mathrm{e}-05$ \\
0.4 & 0.183471 & 0.183281 & $2.9651 \mathrm{e}-05$ & $2.80978 \mathrm{e}-05$ \\
0.5 & 0.213164 & 0.212890 & $3.1178 \mathrm{e}-05$ & $2.95442 \mathrm{e}-05$ \\
0.6 & 0.226510 & 0.226149 & $2.9652 \mathrm{e}-05$ & $2.80986 \mathrm{e}-05$ \\
0.7 & 0.215483 & 0.215063 & $2.5224 \mathrm{e}-05$ & $2.39024 \mathrm{e}-05$ \\
0.8 & 0.172786 & 0.172388 & $1.8326 \mathrm{e}-05$ & $1.73663 \mathrm{e}-05$ \\
0.9 & 0.097316 & 0.097065 & $9.6349 \mathrm{e}-06$ & $9.13007 \mathrm{e}-06$ \\
\hline $\mathrm{L}_{2}$ & $2.5719 \mathrm{e}-04$ & & $1.1549 \mathrm{e}-06$ & \\
\hline
\end{tabular}

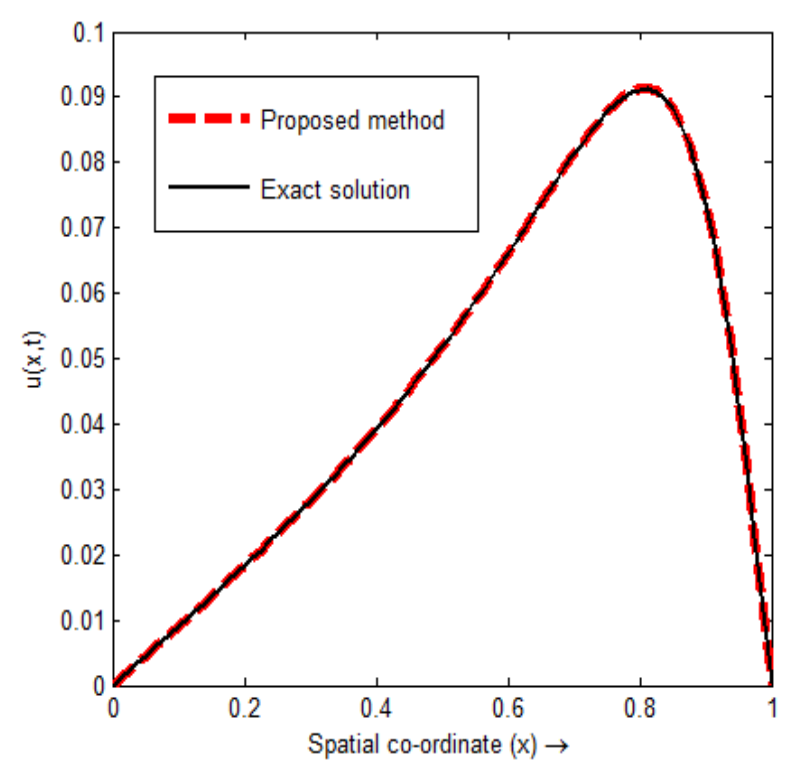

Fig.1. Numerical solution by proposed method and Exact solution for $a=1.1, h=0.0125, T=1, \Delta t=0.001$ and $v=$ 0.01
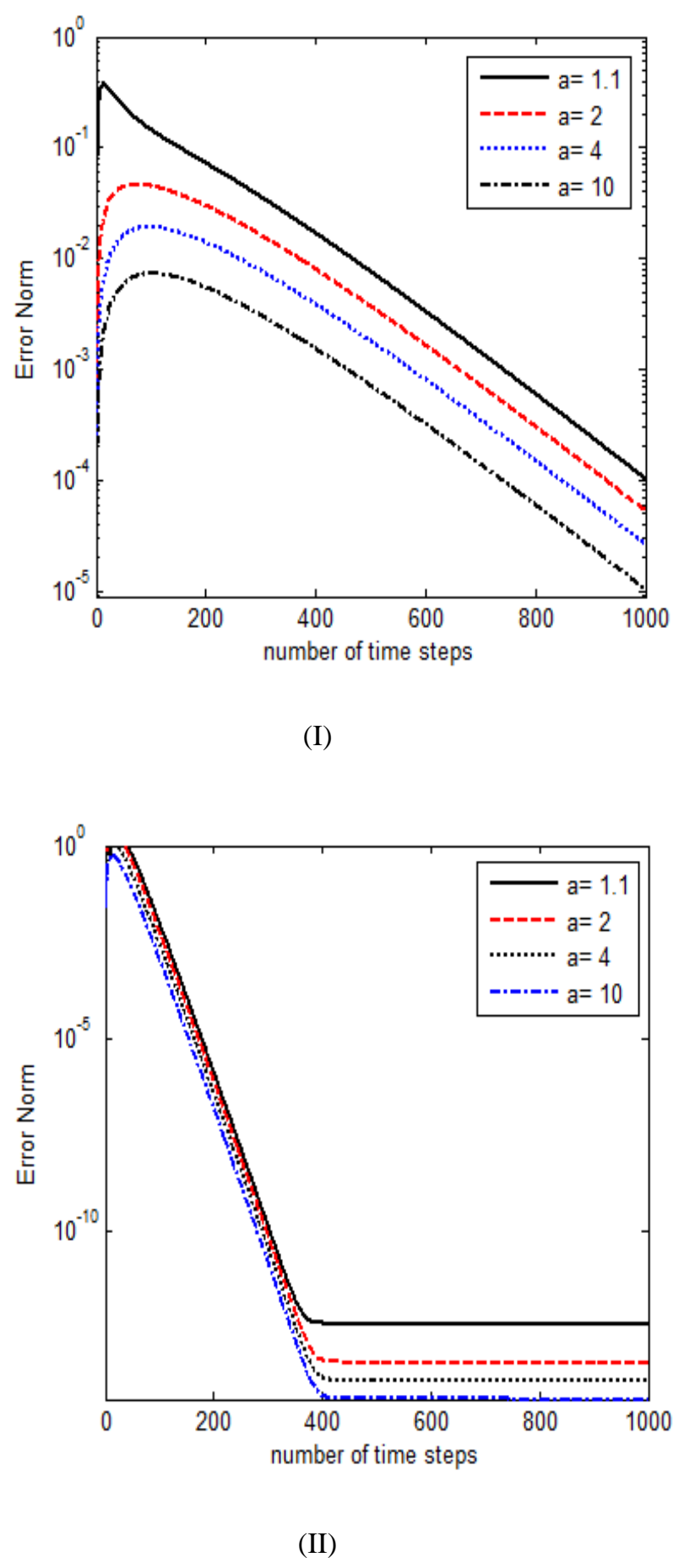

Fig.2. $\mathrm{L}_{2^{-}}$error norm for different values of Burger's coefficient $a$ by using Proposed Method forh $=0.0125, \Delta t=$ $0.001, T=1$ for (I) $v=1$ and (II) $v=10$. 


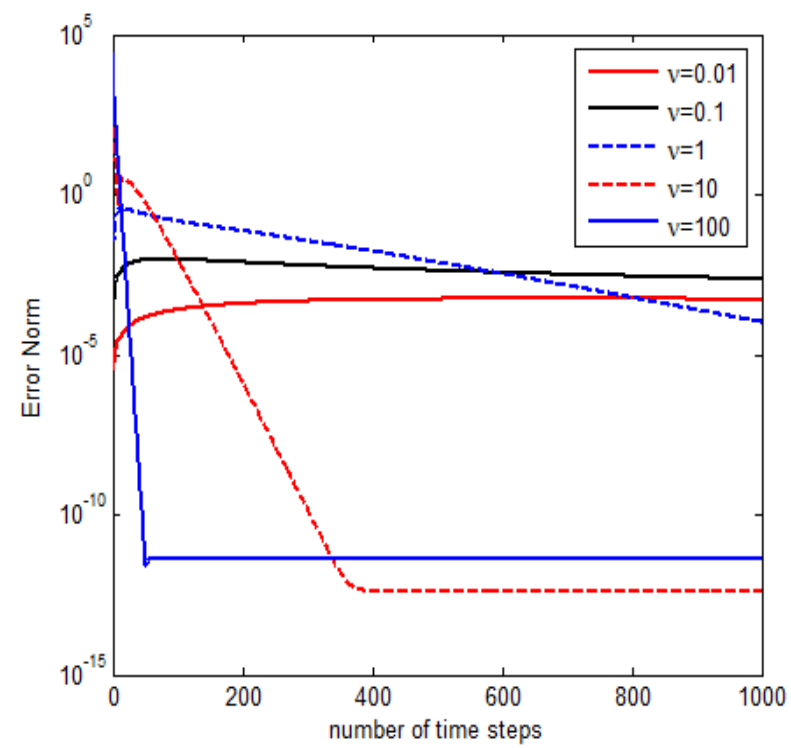

Fig.3. $\mathrm{L}_{2}$ error norm by using proposed method for $a=1.1, h$ $=0.0125, \Delta t=0.001$ at $T=1$ for different value of $v$.

Table 2. Comparison of the proposed method with other known explicit methods to get L2- error norm for $a=1.1, h$ $=0.0125, T=1,10$ for $v=0.001,0.01,0.1$.

\begin{tabular}{|c|c|c|c|c|}
\hline$v$ & $\Delta t$ & $\begin{array}{c}\text { EF } \\
{[35]}\end{array}$ & $\begin{array}{c}\text { MCOR } \\
{[36]}\end{array}$ & $\begin{array}{c}\text { Proposed } \\
\text { Method }\end{array}$ \\
\hline & 0.001 & 3.4891 e-08 & 2.6251 e-08 & 3.4934 e-08 \\
\hline \multicolumn{5}{|l|}{0.0001} \\
\hline & 0.01 & 2.6372 e-07 & 1.9502 e-07 & 2.6729 e-07 \\
\hline & 0.001 & 2.6372 e-06 & 1.9502 e-06 & 2.6729 e-06 \\
\hline \multicolumn{5}{|l|}{0.001} \\
\hline & 0.01 & 4.9467 e-06 & 1.0399 e-05 & 5.9641 e-06 \\
\hline & 0.001 & 4.9467 e-05 & 1.0399 e-04 & 5.9641 e-05 \\
\hline \multicolumn{5}{|l|}{0.01} \\
\hline & 0.01 & Divergence & Divergence & 2.5719 e-05 \\
\hline & 0.001 & Divergence & Divergence & 2.5719 e- 04 \\
\hline \multicolumn{5}{|l|}{0.1} \\
\hline & 0.01 & Divergence & Divergence & 1.1549 e-06 \\
\hline
\end{tabular}

Table 3. L2- error norm at $h=0.0125, \Delta t=0.001, T=1$ for different value of $v$ and $a$.

\begin{tabular}{ccccc}
\hline$A$ & $v=0.1$ & $v=1$ & $v=10$ & $v=100$ \\
\hline 1.1 & $2.5719 \mathrm{e}-04$ & $1.1549 \mathrm{e}-05$ & $4.4842 \mathrm{e}-14$ & $4.4842 \mathrm{e}-13$ \\
2 & $6.8753 \mathrm{e}-05$ & $5.9438 \mathrm{e}-06$ & $4.4842 \mathrm{e}-15$ & $4.4842 \mathrm{e}-14$ \\
4 & $2.7418 \mathrm{e}-05$ & $2.9305 \mathrm{e}-06$ & $1.4947 \mathrm{e}-15$ & $1.4947 \mathrm{e}-14$ \\
\hline
\end{tabular}

Example 2:

Solve Burgers' equation in (1-3) with Initial condition:

$$
u(x, 0)=f(x)=\sin (\pi x)
$$

whose exact solution is given by [5]:

$$
\begin{aligned}
& u(x, t)=f(x) \\
& =2 v \pi \frac{\sum_{n=1}^{\infty} c_{n} \exp \left(-n^{2} \pi^{2} v t\right) n \sin (n \pi x)}{c_{0}+\sum_{n=1}^{\infty} c_{n} \exp \left(-n^{2} \pi^{2} v t\right) \cos (n \pi x)}
\end{aligned}
$$

where the Fourier coefficients are:

$$
\begin{gathered}
c_{0}=\int_{0}^{1} \exp \left\{-\frac{1}{2 v \pi}[1-\cos (\pi x)]\right\} d x \\
c_{n}=2 \int_{0}^{1} \exp \left\{-\frac{1}{2 v \pi}[1-\cos (\pi x)]\right\} \cos (n \pi x) d x
\end{gathered}
$$

Numerical results obtained by the proposed method for $h=0.0125$ and $0.01, T=0.5, v=1$ and time step $\Delta t=0.001$ are listed at different nodal points in Table (4). In Table (5) the comparison of the proposed method with exact solution at different time for $h=0.01, \quad v=0.1$ and time steps $\Delta t=0.01,0.001$ are tabulated. To investigate the stability of the Proposed Method, the $\mathrm{L}_{2}$ - error norms at the last time step are listed in Table (6) for different values of initial condition parameter viscosity $v$.These results show that the proposed method is stable for a wide range of $v$.

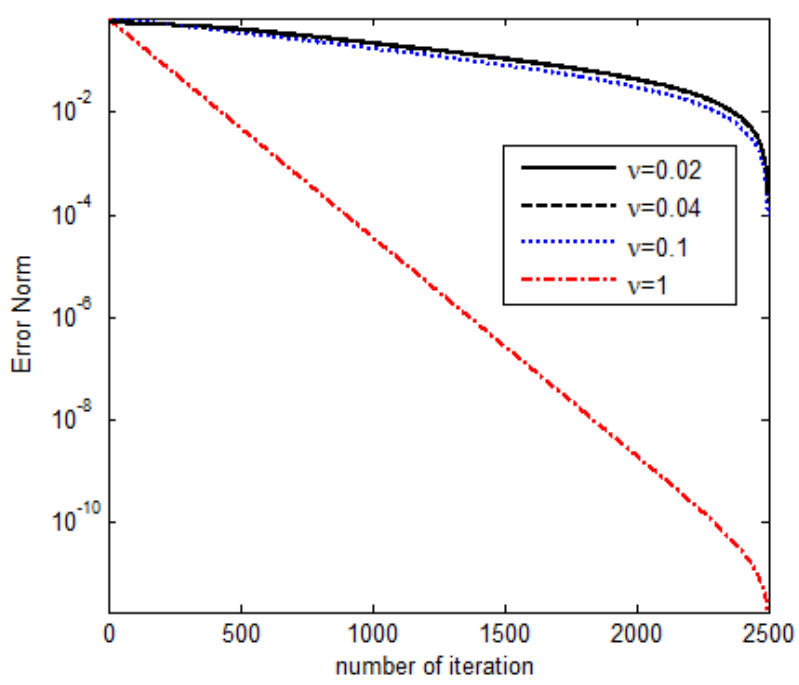

Fig.4. $L_{2^{-}}$error norm for different values of $v$ by using Proposed Method for $h=0.01, \Delta t=0.001, T=2.5$. 
Table 4.Comparison of the proposed method with exact solution at different space points and the $\mathrm{L}_{2}$ - error norm for $h=0.0125$ and $0.01, T=0.5, v=1$ and time step $\Delta t=$ $0.001,0.0001$.

\begin{tabular}{|c|c|c|c|c|c|}
\hline \multirow[t]{2}{*}{$X$} & \multicolumn{3}{|c|}{$\begin{array}{l}\text { Proposed Method } \\
\text { Solution } \\
\Delta t=0.001 \quad \Delta t=0.0001\end{array}$} & Exact & \\
\hline & $M=80$ & $\mathrm{M}=100 \mathrm{~N}$ & $=80$ & 100 & \\
\hline 0.1 & 0.00227 & 0.00227 & 0.00222 & 0.00222 & 0.00221 \\
\hline 0.2 & 0.00432 & 0.00432 & 0.00422 & 0.00422 & 0.00421 \\
\hline 0.3 & 0.00594 & 0.00594 & 0.00581 & 0.00581 & 0.00580 \\
\hline 0.4 & 0.00699 & 0.00699 & 0.00684 & 0.00684 & 0.00682 \\
\hline 0.5 & 0.00735 & 0.00735 & 0.00719 & 0.00719 & 0.00717 \\
\hline 0.6 & 0.00699 & 0.00699 & 0.00684 & 0.00684 & 0.00682 \\
\hline 0.7 & 0.00595 & 0.00595 & 0.00582 & 0.00582 & 0.00580 \\
\hline 0.8 & 0.00432 & 0.00432 & 0.00423 & 0.00423 & 0.00422 \\
\hline 0.9 & 0.00227 & 0.00227 & 0.00223 & 0.00222 & 0.00222 \\
\hline $\mathrm{L}_{2}$ & $1.272 \mathrm{e}-4$ & $1.260 \mathrm{e}-4$ & $1.562 \mathrm{e}-5$ & $1.445 e-5$ & \\
\hline
\end{tabular}

Table 5.Comparison of the proposed method with exact solution at different time for $h=0.01, v=0.1$ and time steps $\Delta t=0.01,0.001$

\begin{tabular}{lllcl}
\hline \multirow{2}{*}{$X$} & $\mathrm{~T}$ & \multicolumn{2}{c}{ Proposed Method } & $\begin{array}{l}\text { Exact } \\
\text { Solution }\end{array}$ \\
\hline \multirow{3}{*}{0.25} & 2.5 & 0.044038 & 0.043459 & 0.043381 \\
& 3.0 & 0.027693 & 0.027261 & 0.027202 \\
& 3.5 & 0.017243 & 0.016932 & 0.016890 \\
\hline \multirow{3}{*}{0.5} & 2.5 & 0.066948 & 0.065988 & 0.065857 \\
& 3.0 & 0.040973 & 0.040296 & 0.040205 \\
& 3.5 & 0.025076 & 0.024608 & 0.024545 \\
\hline \multirow{2}{*}{0.75} & 2.5 & 0.051179 & 0.050374 & 0.050263 \\
& 3.0 & 0.030375 & 0.029844 & 0.029772 \\
& 3.5 & 0.018248 & 0.017896 & 0.017848 \\
\hline
\end{tabular}

Table 6. $\mathrm{L}_{2}$ - error norm at $h=0.01, \Delta t=0.001$ for different value of $v$ and $T$

\begin{tabular}{llll}
\hline \multicolumn{1}{c}{$v$} & $T=1$ & $T=2.5$ & $T=3.0$ \\
\hline 1 & $1.8365 \mathrm{e}-06$ & $1.7740 \mathrm{e}-12$ & $1.5505 \mathrm{e}-14$ \\
0.1 & $2.6350 \mathrm{e}-04$ & $9.4185 \mathrm{e}-05$ & $6.5146 \mathrm{e}-05$ \\
0.04 & $4.8017 \mathrm{e}-04$ & $1.9112 \mathrm{e}-04$ & $1.4670 \mathrm{e}-04$ \\
\hline
\end{tabular}

In Fig. 4, $\mathrm{L}_{2}$ - error norm for different values of $v$ by using Proposed Method for $h=0.01, \Delta t=0.001, T=$ 2.5 are shown. As shown in Fig. 4,v=1 is needed 2500 time iterations to achieve the obtained error norm butdecreasing $v$ doesn't achieve the same rate ofreducing the error norm. This phenomena takes place in case of small viscosity due to appear of a thin layer close to the wall that is known as the boundary layer which is needed to increase grid points into the boundary layer region than the rest of the domain to obtain oscillation-free solutions. Also, small time increment is chosen to ensure high accuracy.

\section{Conclusions}

In this work the new scheme based on finite difference method was proposed to obtain the numerical solution of the 1D-Burgers' equation. The nonlinear term in Burgers' equationlinearized without any transformation formula. The proposed scheme provides accurate solutions of the two test problems, be stable and converge even for low viscosity and long time. Moreover, the proposed scheme is simple and can be to implement easily.

\section{Acknowledgment}

The author is very thankful to the reviewers for their valuable comments and suggestions to improve the quality of the research work.

\section{References}

[1] H. Bateman, Some recent researches on the motion of fluids, Mon. Weather Rev. 43, 163-170,1915.

[2] J.M. Burgers, Mathematical examples illustrating relations occurring in the theory of turbulent fluid motion, NorthHolland Pub. Co.,1-53,1939.

[3] J.M. Burgers, A mathematical model illustrating the theory of turbulence, in: Adv. in Appl. Mech., Vol. I, Academic Press, New York,171-199,1948.

[4] E. Hopf, The partial differential equation $U t+U U_{x}=$ $\mu U_{x x}$,Commun. Pure \& Appl. Math. 3, 201-230, 1950.

[5] J. D. Cole, On a quasi-linear parabolic equation in aerodynamics, Quart. Appl. Math. 9, 225-236, 1951.

[6] M. Ciment, S.H. Leventhal, B.C. Weinberg, The operator compact implicit method for parabolic equations, J. Comput. Phys. 28, 135-166, 1978.

[7] L. Iskandar, A. Mohsen, Some numerical experiments on the splitting of Burgers' equation, Numer. Methods Partial Differential Equations 8, 267-276, 1992.

[8] V. Mukundan, A. Awasthi, Efficient numerical techniques for Burgers' equation, Appl. Math. Comput.262, 282-297, 2015.

[9] T. Ozis, E.N. Aksan, A. Ozdes, A finite element approach for solution of Burgers' equation, Appl. Math. Comput. 139, 417-428, 2003. 
[10] AbdulkadirDogan, A Galerkin finite element approach to Burgers' equation, Appl. Math. Comput. 157, 331-346, 2004.

[11] B. De Maerschalck, M.I. Gerritsma, The use of Chebyshev polynomials in the space-time least-squares spectral element method, Numer. Alg. 38, 173-196, 2005.

[12] W. Heinrichs, An adaptive spectral Least-Squares scheme for the Burgers equation, Numer. Alg. 44, 1-10, 2007.

[13] B. De Maerschalck, M.I. Gerritsma, Least-squares spectral element method for non-linear differential equations, J. Comput. Appl. Math. 215, 357-367, 2008.

[14] A.H.A. Ali, G.A. Gardner, L.R.T. Gardner, A collocation solution for Burgers' equation using cubic B-spline finite elements, Comput. Methods Appl. Mech. Eng. 100, 325-337, 1992.

[15] R.C. Mittal, R.K. Jain, Numerical solutions of nonlinear Burgers' equation with modified cubic B-splines collocation method, Appl. Math. Comput. 218, 7839-7855, 2012.

[16] B. Saka, I. Dag, Quartic B-spline collocation method to the numerical solutions of the Burgers' equation, Chaos, Solitons and Fractals 32, 1125-1137, 2007.

[17] S. Kutulay, A.R. Bahadir, A. Odes, Numerical solution of the one-dimensional Burgers' equation: explicit and exactexplicit finite difference methods, J. Comput. Appl. Math. 103, 251-261, 1999.

[18] M. A. Abdou, A.A. Soliman, Variational iteration method for solving Burgers and coupled Burgers equations, J. Comput. Appl. Math. 181, 245-251, 2005.

[19] J. Biazar, H.Aminikhah, Exact and numerical solutions for non-linear Burger's equation by VIM, Mathematical and Computer Modelling 49, 1394-1400, 2009.

[20] M. Dehghan, A. Hamidi, M. Shakourifar, The solution of coupled Burgers equations using Adomian-Pade technique, Appl. Math. Comput. 189, 1034-1047, 2007.

[21] M.M. Rashidi, G. Domairry, S. Dinarvand, Approximate solutions for the Burgers' and regularized long wave equations by means of the homotopy analysis method, Commun. Nonlinear Sci. Numer. Simul. 14, 708-717, 2009.

[22] M.M. Rashidi, E. Erfani,Newanalytic method for solving Burgers' and nonlinear heat transfer equations and comparison with HAM, Comput. Phys. Comm. 180, 15391544, 2009.

[23] A.H. Khater, R.S. Temsah, M.M. Hassan, A Chebyshev spectral collocation method for solving Burgers' type equations, J. Comput. Appl. Math. 222, 333-350, 2008.
[24] A.K. Khalifa, KhalidaInayat Noor, Muhammad Aslam Noor, Some numerical methods for solving Burgers equation, Int. J. Phys. Sci. 6, 1702-1710, 2011.

[25] A. Korkmaz, Idris Dag, Polynomial based differential quadrature method for numerical solution of nonlinear Burgers’ equation, J. Franklin Inst. 348, 2863-2875, 2011.

[26] R.C. Mittal, R.K. Jain, Numerical solutions of nonlinear Burgers' equation with modified cubic B-splines collocation method, Appl. Math. Comput. 218, 7839-7855, 2012.

[27] G. Arora, B. K. Singh, Numerical solution of Burgers' equation with modified cubic B spline differential quadrature method, Appl. Math. Comput. 224, 166-177, 2013.

[28] M. Tamsir, V. Srivastava, R. Jiwari, An algorithm based on exponential modified cubic B spline differential quadrature method for nonlinear Burgers' equation, Appl. Math. Comput. 290, 111-124, 2016.

[29] I.A. Ganaie, V.K. Kukreja, Numerical solution of Burgers' equation by cubic Hermite collocation method, Appl. Math. Comput. 237, 571-581, 2014.

[30] R. Jiwari, A hybrid numerical scheme for the numerical solution of the Burgers' equation, Comput. Phys. Comm. 188, 59-67, 2015.

[31] M. Seydaoğlu, U. Erdoğan , T. Öziş , Numerical solution of Burgers' equation with high order splitting methods, Comput. Appl. Math. 291, 410-421, 2016.

[32] T. Zhanlav , O. Chuluunbaatar , V. Ulziibayar, Higher-order accurate numerical solution of unsteady Burgers' equation, Appl. Math. Comput. 250, 701-707, 2015.

[33] D. A. Kay, P. M. Gresho, D. F. Griffiths and D. J. Silvester, Adaptive time-stepping for incompressible flow Part II: Navier-Stokes Equations, Siam J. Sci. Comput.32, No. 1, 111-128, 2010.

[34] W.L. Wood, An exact solution for Burger's equation, Commun. Numer. Meth. Eng. 22, 797-798, 2006.

[35] T. Bui, Explicit and Implicit Methods In Solving Differential Equations, University of Connecticut , Honors Scholar Theses, 119, 2010.(http://digitalcommons.uconn.edu/srhonors_theses/119)

[36] A. Perrin, H. H. Hu, An Explicit Finite-Difference Scheme for Simulation of Moving Particles, J. Comput. Phys., 212, 166-187, 2006. 